\title{
Association between Thyroid Autoimmunity and Fibromyalgia
}

Authors

Affiliations

\section{J. H. Suk ${ }^{1}$, J. H. Lee ${ }^{2}$, J. M. Kim ${ }^{3}$}

Department of Internal Medicine, Division of Endocrinology and Metabolism, Maryknoll Medical Center, Busan, Republic of Korea

Department of Internal Medicine, Division of Rheumatology, Maryknoll Medical Center, Busan, Republic of Korea Department of Internal Medicine, Division of Endocrinology and Metabolism, Sanggye Paik Hospital, Inje University College of Medicine, Seoul, Republic of Korea

\author{
Key words \\ - thyroid \\ autoimmunity \\ - Fibromyalgia
}

received $\quad 02.12 .2011$

first decision 26.01.2012

accepted 13.03.2012

Bibliography

DOI http://dx.doi.org/

10.1055/s-0032-1309008

Published online:

April 27, 2012

Exp Clin Endocrinol Diabetes

2012; 120: 401-404

(c) J. A. Barth Verlag in

Georg Thieme Verlag KG

Stuttgart · New York

ISSN 0947-7349

\section{Correspondence}

\section{J. H. Lee, MD}

Department of Internal Medicine

Division of Rheumatology Maryknoll Medical Center

4-12, Daecheong-dong

Jung-gu

Busan

Korea

Tel.: +82/52/461 2475

Fax: $+82 / 51 / 4657470$

ete@lycos.co.kr

\section{Abstract}

$\nabla$

Background and aims: Evidence exists that autoimmune thyroiditis is present in a high percentage of fibromyalgia (FM) and associated with the presence of typical symptoms of FM. However, the role of thyroperoxidase antibody (TPO $\mathrm{Ab}$ ) in the manifestation of FM is still unclear. The goal of this study was to investigate the prevalence of positive TPO Ab in euthyroid FM patients, and whether TPO Ab positivity is associated with the clinical manifestations in euthyroid FM patients.

Subjects and Methods: Thyroid assessment was done by free T4, TSH and TPO Ab. The clinical parameters including Fibromyalgia Impact questionnaire (FIQ), pain visual analogical scale (VAS) and tender point counts were evaluated in euthyroid primary FM patients, not associated with autoimmune rheumatic disease. The immunologic tests including rheumatoid factor and antinuclear antibody were measured. We compared the prevalence of positive TPO Ab between FM

\section{Introduction \\ $\nabla$}

Chronic autoimmune thyroiditis, also called Hashimoto's thyroiditis (HT), is the most common and extensively studied organ-specific autoimmune disorder in human (Dayan and Daniels, 1996). Patients with autoimmune thyroid disorders, more often HT but also Graves' disease, often have rheumatic manifestations including a mild non-erosive variety or arthritis, polyarthralgia, myalgia, and sicca syndrome without a true Sjogren's syndrome. 2 possibilities can be considered to explain rheumatic manifestations, associated with HT with euthyroid state. The first, rheumatic manifestations could be attributable to some autoimmune rheumatic diseases associated or overlapping with HT, such as rheumatoid patients, and healthy control. We also compared clinical and laboratory parameter in FM patients according to the presence of TPO Ab.

Results: 149 patients of FM, 68 healthy controls were recruited. FM patients showed higher prevalence of positive TPO Ab than healthy controls ( 28 out of 149 patients, $19 \%$; 5 out of 68 healthy controls, $7 \%$; $\mathrm{P}=0.04)$. There was no difference of clinical and laboratory parameters in FM patients between 2 groups subdivided by the presence of TPO Ab.

Conclusion: In our study, euthyroid FM patients showed significantly higher prevalence of positive TPO Ab, as compared to age and sex matched healthy control. However, TPO Ab positivity was relatively low and not associated with the clinical manifestations in euthyroid FM patients. This finding support thyroid autoimmunity may influence the development of FM, but the evidence which support that FM is related to autoimmune etiology is not clear, and FM severity may not be affected by the presence of thyroid autoantibody.

arthritis, systemic lupus erythematosus (SLE), Sjogren's syndrome, or scleroderma (Punzi and Betterle, 2004). The other possibility is that some rheumatic manifestations are related to underlying thyroid autoimmunity.

Fibromyalgia (FM) is a commonly encountered disorder characterized by chronic widespread musculoskeletal pain and related symptoms along with multiple painful tender points (Buskila and Sarzi-Puttini, 2006). FM is a frequent secondary manifestation of many autoimmune rheumatic diseases. But primary FM is not generally regarded as an autoimmune rheumatic disease. Recent studies revealed that primary FM patients showed high prevalence of thyroid autoantibodies similar to that of above-mentioned autoimmune rheumatic diseases (Bazzichi et al., 2007; 
Pamuk and Cakir, 2007). Though this high prevalence of thyroid autoantibodies in FM patients is not fully explained, possible mechanism is a direct association with thyroid autoimmunity. We performed this study to investigate the prevalence of positive thyroperoxidase antibody (TPO Ab) in patients with euthyroid primary FM, compared to healthy control and whether TPO $\mathrm{Ab}$ positivity is associated with the clinical manifestations in euthyroid FM patients.

\section{Methods \\ $\nabla$ \\ Patients}

FM patients were recruited at the Division of Rheumatology in Maryknoll Medical Center from February 2009 to June 2010. Diagnosis of FM was established by criteria according to the American College of Rheumatology (ACR), which included pain for more than 3 months from all of the 4 body quadrants, axial skeletal pain, and pain upon digital palpation of at least 11 out of 18 specific bilateral points (Wolfe et al., 1990). FM Patients with autoimmune rheumatic diseases, which included patients with ANA titers above 1:160, were excluded. Patients with past history of thyroid diseases, palpable goiter, treatment with lithium or amiodarone, or neoplastic disease were also excluded. Patients with abnormal free T4 and/or TSH level were also excluded. Healthy control group was recruited from age and sex matched euthyroid subjects without past history of thyroid diseases and palpable goiter, who had visited the healthy promotion center at Maryknoll Medical Center. We compared the prevalence of positive TPO Ab in FM patients vs. healthy controls. We also compared clinical and laboratory parameter in FM patients according to the presence of TPO Ab. This study was approved by the local research ethics committee, and informed consent was obtained from all participants.

\section{Clinical parameters}

We evaluated general clinical parameters of all recruited patients including age, gender and menopausal status. More detailed clinical parameters including fibromyalgia impact questionnaire (FIQ), pain visual analogical scale (VAS) and tender point (TP) counts were evaluated in FM patients. Patients with FM were asked to rate their current level of pain on the $10 \mathrm{~cm}$ VAS $(0=$ no pain, $10=$ worst pain imaginable) for describing pain intensity at the rheumatology visit. Subjects then were asked to fill out the FIQ that evaluates physical function, work status, depression, anxiety, sleep, pain, stiffness, fatigue, and well being status (Bae and Lee, 2004). The FIQ is a validated, self-administered inventory that correlates with the degree of disability (Abeles et al., 2008). Higher scores indicate a greater impact of the FM syndrome on the patient. Subjects were then assessed for the number of positive TP by digital palpation over the 18 characteristic tender point sites according to ACR diagnostic criteria of FM. The subjects were asked to identify if a given point was painful as slow steady digital pressure was applied. Tender point count was determined by the number of tender points that had a threshold of $4 \mathrm{~kg} / \mathrm{cm}^{2}$. The total TP score (sum of right plus left) was used in the statistical analysis.

\section{Laboratory tests}

Thyroid function was assessed by determining free T4 (FT4) and thyroid stimulating hormone (TSH). FT4 (reference range 9.16$24.9 \mathrm{pmol} / \mathrm{L}$ ) and TSH (reference range $0.38-4.7 \mathrm{mIU} / \mathrm{L}$ ) was measured by chemiluminescent microparticle immunoassay (Architect-I 2000, Abbot, Ireland, UK). TPO Ab was measured by chemiluminescent immunoassay (ADVIA centaur, Germany, Siemens). Cut-off value of TPO Ab was above $60 \mathrm{IU} / \mathrm{ml}$. Highly sensitive $C$ reactive protein (hsCRP), $R A$ factor and antinuclear antibody (ANA) were also measured. hsCRP (reference range $0-0.5 \mathrm{mg} / \mathrm{L}$ ) and RA factor (reference range $015 \mathrm{IU} / \mathrm{ml}$ ) was measured by turbidometric immunoassay (ADVIA1800, Germany, Siemens), and ANA was measured by indirect fluorescent assay.

\section{Statistical analysis}

Continuous variables are expressed as means \pm standard deviations. Statistical comparisons were performed with Student's t-test or Fisher's exact test, as appropriate. Calculations were performed using R statistics software (R Development Core Team, 2010). A P-value $<0.05$ was considered statistically significant.

\section{Results}

$\nabla$

\section{Clinical and laboratory parameters of FM patients in} comparison with healthy controls ( $\bullet$ Table 1 )

149 patients of FM, and 68 healthy controls were included in this study. In FM patients, the mean age was $51 \pm 9$ year (27-71), and $93 \%$ (139 patients) of them was women. Of these women, $64 \%$ (82 out of 129 patients) was post-menopause. ANA was positive (titer < $1: 160$ ) in $29 \%$ (39 out of 134 patients and RA factor was positive in $10 \%$ ( 14 out of 149 patients). Their mean duration of disease was 5 years ( 0 Table 2 ). All of the patients were

\begin{tabular}{|c|c|c|c|}
\hline & $\begin{array}{l}\text { FM } \\
(n=149)\end{array}$ & $\begin{array}{l}\text { control } \\
(n=68)\end{array}$ & $\begin{array}{l}\text { P-values } \\
\text { (FM vs. control) }\end{array}$ \\
\hline age (yr) & $51.2 \pm 8.7$ & $52.2 \pm 8.1$ & 0.387 \\
\hline female, $n(\%)$ & $139(93)$ & $61(90)$ & 0.416 \\
\hline post-menopause, n (\%) & $82 / 129(64)$ & $31 / 42(74)$ & 0.896 \\
\hline positive antinuclear antibody, $\mathrm{n}(\%)$ & 39/134 (29) & $10 / 35(29)$ & 1 \\
\hline RA factor & $12.1 \pm 20.3$ & $9.0 \pm 9.2$ & 0.179 \\
\hline positive RA factor, n (\%) & $14 / 149(9.7)$ & $3 / 38(7.9)$ & 1 \\
\hline hsCRP & $0.9 \pm 3.0$ & $1.0 \pm 3.0$ & 0.911 \\
\hline positive TPOAb, n (\%) & $28(18.8)$ & $5(7.4)$ & 0.04 \\
\hline free T4 (pmol/L) & $13.5 \pm 2.1$ & $14.2 \pm 2.0$ & 0.03 \\
\hline $\mathrm{TSH}(\mathrm{mlU} / \mathrm{L})^{*}$ & $1.58 \pm 1.70$ & $1.47 \pm 1.81$ & 0.417 \\
\hline
\end{tabular}

hsCRP, highly sensitive CRP; TPOAb, thyroperoxidase antibody; * TSH values were shown as geometric means and geometric standard deviations

Table 1 Clinical characteristics of fibromyalgia (FM) patients in comparison with healthy controls. 
euthyroid, which was an inclusion criterion. There was no difference of age, sex, menopausal status, positivity of ANA and RA factor, hsCRP titers in FM patients vs. healthy controls. FM patients showed higher prevalence of positive TPO Ab than healthy controls ( 28 out of 149 patients, $19 \%$; 5 out of 68 healthy controls, 7\%; $\mathrm{P}=0.04$ ). Distributions of TPO Ab titers in FM patients and healthy controls are shown in $\bullet$ Fig. 1.

\section{Comparisons of clinical and laboratory parameters in FM patients according to the presence of TPO Ab (o Table 2)}

We compared clinical and laboratory parameters in FM patients between 2 groups subdivided by the presence of TPO Ab. TPO Ab was positive in 28 out of $149 \mathrm{FM}$ patients. All patients with positive TPO $\mathrm{Ab}$ and $92 \%$ of patients with negative TPO Ab were women. There was no difference in regards to age, sex, menopausal status, and disease duration of FM between 2 groups. FIQ TP counts, and pain VAS did not show any difference between 2 groups. TPO Ab titiers showed no correlation with FIQ $(P=0.934)$, which indicates TPO Ab titers had no impact on the degree of disability of FM. Pain parameters, such as pain VAS and TP counts, also demonstrated no correlation with TPO Ab titers $(P=0.951,0.462$, respectively). There was also no difference in ANA positivity, RA factor positivity, hsCRP, free T4 and TSH between 2 groups. The study participants were also subdivided into 2 groups based on the sum of the FIQ score (group A: FIQ $\geq 50$, group B: $<50$ ). We compared TPO Ab positivity in FM patients between 2 groups. But, there was no difference in prevalence of TPO Ab between 2 groups (group A: 15 out of 85 patients, $18 \%$; group B: 14 out of 64 patients, $22 \% ; P=0.672$ ).

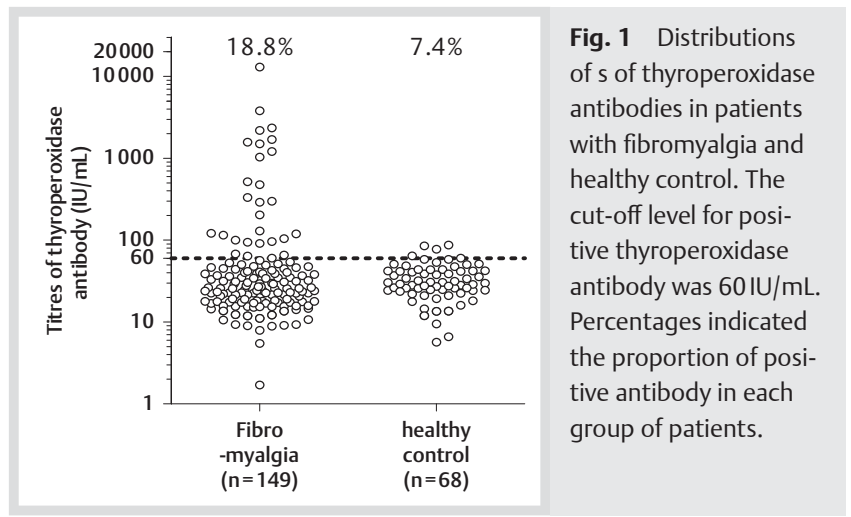

\section{Discussion \\ $\nabla$}

Autoimmune diseases are relatively common and have often non-specific clinical manifestations, especially early in the disease, making diagnosis difficult. In such patients, the presence of autoantibodies may be useful diagnostic markers. An autoantibody may be considered pathogenic if 1 ) it is present in concentrations significantly higher and more frequently than in a control population; 2 ) it is specifically directed against a pathologically relevant autoantigen; and 3 ) the disease is reproducible by the injection or induction of the autoantibody in experimental animals (D'Cruz 2002). HT is the most common and extensively studied organ-specific autoimmune disorder in human (Dayan and Daniels, 1996). HT is characterized by diffuse lymphocytic infiltration of the thyroid gland, presence of TPO $\mathrm{Ab}$ and/or Thyroglobulin $\mathrm{Ab}(\mathrm{Tg} \mathrm{Ab})$ in serum, clinical evidence of goitrous or atrophic gland, and frequent thyroid dysfunction of varying degrees ${ }^{1}$ (Dayan and Daniels, 1996). It is well-known that TPO Ab is closely associated with overt thyroid dysfunction, and its presence tends to correlate with thyroid damage and lymphocytic inflammation (Pearce et al., 2003).

FM is a condition of chronic widespread musculoskeletal pain and tenderness, characterized by hyperalgesia (heightened sensitivity to noxious stimuli), and allodynia (nonnoxious stimuli may result in pain) (Buskila and Sarzi-Puttini, 2006). Though the pathogenesis of FM is not entirely understood, the current concept is regarded as the result of central nervous system malfunction resulting in amplification of pain transmission and interpretation (Buskila and Sarzi-Puttini, 2006). It has long been recognized that there are significant similarities between the clinical findings in FM and symptoms of thyroid dysfunction (Bland and Frymoyer, 1970). Many studies reported that a FM patients had some problems in the production or utilization of thyroid hormones (Garrison and Breeding, 2003). Studies recently reported that there was an association between thyroid autoimmunity and FM (Bazzichi et al., 2007; Pamuk and Cakir, 2007; Ribeiro and Proietti, 2004).

Muscle symptoms are frequent in thyroid diseases and are usually related to hypothyroidism or hyperthyroidism (Khaleeli et al., 1983). However, some recent reports have found clinical and biochemical features of muscle dysfunction even in subclinical hypothyroidism (Monzani et al., 1997). In population studies, it was observed that the prevalence of thyroid autoantibodies was higher in subjects with musculoskeletal complaints (Aarflot and Bruusgaard, 1996). It has been suggested that the presence

Table 2 Clinical characteristics of fibromyalgia (FM) patients according to the presence of thyroperoxidase antibody (TPO Ab).

\begin{tabular}{|c|c|c|c|c|}
\hline & Positive TPO Ab $(n=28)$ & Negative TPO Ab $(n=121)$ & P-values ${ }^{a}$ & Overall $(n=149)$ \\
\hline age (yr) & $53.4 \pm 8.3$ & $50.7 \pm 8.8$ & 0.135 & $51.2 \pm 8.7$ \\
\hline female, $\mathrm{n}(\%)$ & $28(100)$ & $111(92)$ & 0.209 & $139(93)$ \\
\hline post-menopause, n (\%) & $18 / 26(69)$ & $64 / 103(62)$ & 0.649 & $82 / 129(64)$ \\
\hline duration of disease (yr) & $5.0 \pm 5.2$ & $5.1 \pm 6.0$ & 0.952 & $5.1 \pm 5.8$ \\
\hline fibromyalgia Impact questionnaire & $47.2 \pm 18.1$ & $47.9 \pm 18.2$ & 0.856 & $47.8 \pm 18.1$ \\
\hline tender point counts & $13.6 \pm 1.8$ & $13.4 \pm 1.8$ & 0.633 & $13.4 \pm 18.1$ \\
\hline pain visual analogical scale & $51.8 \pm 20.0$ & $54.0 \pm 21.8$ & 0.604 & $53.6 \pm 21.4$ \\
\hline positive antinuclear antibody, $\mathrm{n}(\%)$ & $11 / 24(46)$ & $28 / 110(25)$ & 0.08 & 39/134 (29) \\
\hline RA factor & $11.8 \pm 15.6$ & $12.2 \pm 21.2$ & 0.911 & $12.1 \pm 20.3$ \\
\hline hsCRP & $0.8 \pm 1.2$ & $1.0 \pm 3.3$ & 0.568 & $0.9 \pm 3.0$ \\
\hline free T4 (pmol/L) & $14.1 \pm 2.0$ & $13.4 \pm 2.0$ & 0.111 & $13.6 \pm 2.0$ \\
\hline $\mathrm{TSH}(\mathrm{mlU} / \mathrm{L})^{*}$ & $1.59 \pm 1.86$ & $1.57 \pm 1.67$ & 0.91 & $1.58 \pm 1.70$ \\
\hline
\end{tabular}

a Positive vs. negative TPOAb; hsCRP, highly sensitive CRP; * TSH values were shown as geometric means and geometric standard deviations 
of muscle symptoms in euthyroid subjects with HT might be associated with pre-subclinical hypothyroidism (Ribeiro and Proietti, 2004). An electron microscopic study of skeletal muscle biopsies from euthyroid and hypothyroid patients with HT showed the existence of capillary alterations and a mononuclear cell infiltrate (Marquez et al., 2001).

In our study, $19 \%$ of 149 primary FM patients (mean 51 years) exhibited positive TPO Ab, which was significantly higher compared to that of control group (7\%). It is known that about $10 \%$ in healthy people have TPO Ab although it may reach $30 \%$ in the elderly (Mariotti et al., 1990; Pedersen et al., 2003). Thyroid antibody prevalence rates are difficult to compare because different biochemical methods and study designs have been applied, and age and sex composition of studies differs widely. In addition, most previous studies was done in populations with iodine deficiency, and there is not much known about prevalence of thyroid antibodies in iodine-sufficient area like our country. Even considering the points that previous studies demonstrated similarly higher prevalence of thyroid autoantibodies in FM patients (Bazzichi et al., 2007; Pamuk and Cakir, 2007), and we evaluated prevalence of TPO Ab in euthyroid FM patients using strict criteria, this prevalence seems to be relatively low. Therefore, referring to FM as an autoimmune disease seems to be speculative at the present time.

There was no difference as regards FIQ TP, and pain VAS between FM patients with TPO Ab and those without TPO Ab. Also, other authors did not find any differences in the FIQ scores between FM patients with or without thyroid autoimmunity (Bazzichi et al., 2007; Pamuk and Cakir, 2007). The association between thyroid autoimmunity and fibromyalgic disease severity were not consistently reported in previous studies (Bazzichi et al., 2007; Pamuk and Cakir, 2007). Some studies reported relationship between thyroid autoimmunity and several symptoms like allodynia, dry eyes, sore throat, blurred vision, tension headache or depression (Bazzichi et al., 2007; Pamuk and Cakir, 2007; Ribeiro and Proietti, 2004). Sore throat, blurred vision, and dry eyes are symptoms that may be related to a subclinical thyroid disease. Because we did not evaluated individual symptoms in FM patients, we cannot comment on this on the present.

In this study, euthyroid FM patients showed significantly higher prevalence of positive TPO $\mathrm{Ab}$, as compared to age and sex matched healthy control. However, TPO Ab positivity was relatively low and not associated with the clinical manifestations in euthyroid FM patients. This finding support thyroid autoimmunity may influence the development of FM, but the evidence which support that FM is related to autoimmune etiology is not clear, and FM severity may not be affected by the presence of thyroid autoantibody. Prospective studies with large number of FM patients and euthyroid patients with HT are needed, to understand this association.

\section{References}

1 Aarflot T, Bruusgaard D. Association between chronic widespread musculoskeletal complaints and thyroid autoimmunity. Results from a community survey: Scand J Prim Health Care 1996; 14: 111-115

2 Abeles M, Solitar BM, Pillinger $M H$ et al. Update on fibromyalgia therapy. Am J Med 2008; 121: 555-561

3 Bae SC, Lee JH. Cross-cultural adaptation and validation of the Korean fibromyalgia impact questionnaire in women patients with fibromyalgia for clinical research. Qual Life Res 2004; 13: 857-861

4 Bazzichi L, Rossi A, Giuliano $T$ et al. Association between thyroid autoimmunity and fibromyalgic disease severity. Clin Rheumatol 2007; 26: 2115-2120

5 Bland JH, Frymoyer JW. Rheumatic syndromes of myxedema. N Engl J Med 1970; 282: 1171-1174

6 Buskila D, Sarzi-Puttini P. Biology and therapy of fibromyalgia. Genetic aspects of fibromyalgia syndrome: Arthritis Res Ther 2006; 8: 218

7 D'Cruz D. Testing for autoimmunity in humans. Toxicol Lett 2002; 127: $93-100$

8 Dayan CM, Daniels GH. Chronic autoimmune thyroiditis. N Engl J Med 1996; 335: 99-107

9 Garrison RL, Breeding PC. A metabolic basis for fibromyalgia and its related disorders: the possible role of resistance to thyroid hormone: Med Hypotheses 2003; 61: 182-189

10 Khaleeli AA, Griffith DG, Edwards RH. The clinical presentation of hypothyroid myopathy and its relationship to abnormalities in structure and function of skeletal muscle. Clin Endocrinol (Oxf) 1983; 19: $365-376$

11 Mariotti S, Caturegli P, Piccolo P et al. Antithyroid peroxidase autoantibodies in thyroid diseases. J Clin Endocrinol Metab 1990; 71: 661-669

12 Marquez A, Finol HJ, De Blanco MC et al. Skeletal muscle microvascular alterations in euthyroid and hypothyroid patients with autoimmune thyroid disease. J Submicrosc Cytol Pathol 2001; 33: 425-432

13 Monzani F, Caraccio N, Siciliano G et al. Clinical and biochemical features of muscle dysfunction in subclinical hypothyroidism. J Clin Endocrinol Metab 1997; 82: 3315-3318

14 Pamuk ON, Cakir N. The frequency of thyroid antibodies in fibromyalgia patients and their relationship with symptoms. Clin Rheumatol 2007; 26: 55-59

15 Pearce EN, Farwell AP, Braverman LE. Thyroiditis: N Engl J Med 2003; 348: 2646-2655

16 Pedersen IB, Knudsen N, Jorgensen $T$ et al. Thyroid peroxidase and thyroglobulin autoantibodies in a large survey of populations with mild and moderate iodine deficiency. Clin Endocrinol (Oxf) 2003; 58: $36-42$

17 Punzi L, Betterle C. Chronic autoimmune thyroiditis and rheumatic manifestations. Joint Bone Spine 2004; 71: 275-283

18 R Development Core Team. R: A language and environment for statistical computing. Vienna, Austria: R Foundation for Statistical Computing, 2010; URL http://www.R-project.org

19 Ribeiro LS, Proietti FA. Interrelations between fibromyalgia, thyroid autoantibodies, and depression. J Rheumatol 2004; 31: 2036-2040

20 Wolfe F, Smythe HA, Yunus MB et al. The American College of Rheumatology 1990 Criteria for the Classification of Fibromyalgia. Report of the Multicenter Criteria Committee: Arthritis Rheum 1990; 33: $160-172$ 